Trauma Berufskrankh 2010 - 12 [Suppl 4]:472-474 DOI 10.1007/s10039-010-1639-2

Online publiziert: 15 . September 2010

(c) Springer-Verlag 2010

\author{
A.H. Tiemann ${ }^{1} \cdot$ M. Steen ${ }^{2} \cdot$ G.O. Hofmann $n^{3,4}$ \\ ${ }^{1}$ Abteilung für Septische und Rekonstruktive Chirurgie, Klinik für Unfall- \\ und Wiederherstellungschirurgie , BG-Kliniken Bergmannstrost Halle, Saale \\ ${ }^{2}$ Klinik für Plastische und Handchirurgie/Brandverletztenzentrum, \\ BG-Kliniken Bergmannstrost Halle, Saale \\ ${ }^{3}$ Klinik für Unfall- und Wiederherstellungschirurgie, \\ BG-Kliniken Bergmannstrost Halle, Saale \\ ${ }^{4}$ Kliniken für Unfall-, Hand- und Wiederherstellungschirurgie, \\ Friedrich-Schiller-Universität Jena
}

\section{Infektsanierung und Defektdeckung} Algorithmus und Reihenfolge -
chirurgische Strategie

sen können. Grundsätzlich sind hierbei endo- und exogene Faktoren zu unterscheiden $[4,5]$.

\section{Endogene Faktoren [3].}

- lokale Faktoren (Z. B. Frakturausmaß, -lokalisation usw.)

- systemische Faktoren [Z. B. Begleiterkrankungen wie HIV-Infektion („human immunodeficiency virus") oder Rheuma]

\section{Exogene Faktoren.}

- Inokulation von Keimen nach Trauma oder chirurgischen Maßnahmen

- "therapeutischer Effekt" (positive und negative Beeinflussung des Heilveroben genannten Forderungen beeinflus-

Will man diese Ziele realisieren, muss man sich der breiten Palette von Faktoren bewusst sein, die die Umsetzung der

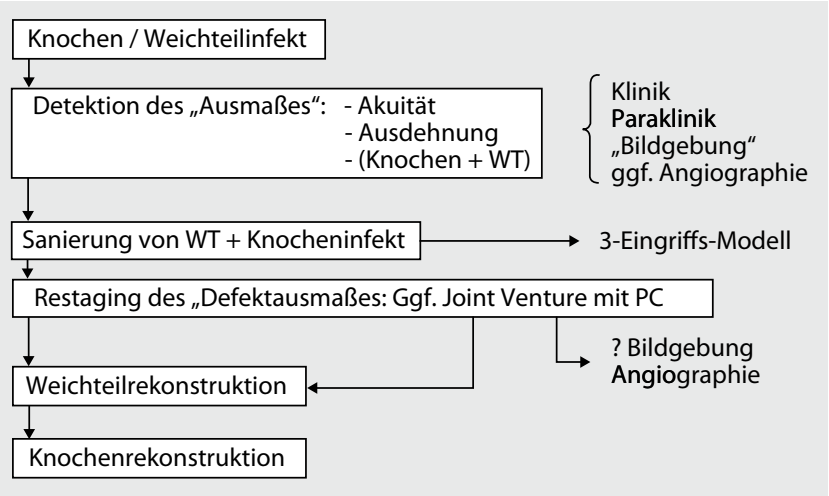

Abb. 14 Algorithmus für die Behandlung eines Knocheninfekts, WT Weichteile laufs durch die einzelnen Behandlungsmaßnahmen)

- Beginn der Infektbehandlung

Gerade Letzteres spielt eine wesentliche Rolle, da seit langem bekannt ist, dass die Inokulation von Keimen innerhalb von kürzester Zeit (Staphylococcus aureus innerhalb von Stunden) irreversible Veränderungen am Knochen hervorruft. Insofern sind die frühzeitige Diagnose und eine sich daran anschließende frühzeitige Therapie die Grundlage für eine erfolgreiche Behandlung [6]. Sie basiert auf 3 Säulen:

1. konsequente chirurgische Infektsanierung

2. Kenntnis und Einsatz von Antibiotika

3. Kenntnis und Einsatz adjuvanter Maßnahmen

\section{Infektbehandlung}

\section{Schritt 1: Chirurgische Infektsanierung}

Die konsequente chirurgische Infektsanierung stellt unverändert die Basis jedweder Behandlung von Knochen- und Gelenkinfektionen dar (• Abb. 1). Daran hat auch die Entwicklung neuer Anti- 
biotika nichts geändert. Wesentlich für die korrekte Durchführung dieser Operationen ist, dass nicht nur die infizierten Knochenareale entfernt werden müssen, sondern ebenso die infizierten umgebenden Weichteile, und zwar ohne Rücksicht auf den Typ des infizierten Weichgewebes (Sehnen, Muskeln, Gefäße, Nerven). In Anlehnung an die onkologische Chirurgie ist eine Ro-Resektion anzustreben. Nur im Frühstadium eines Knocheninfekts kann unter bestimmten Voraussetzungen (geringe Infektausdehnung an Knochen und Weichteilen, Titanimplantate, fester Sitz des implantierten Materials) der Erhalt einer vorhandenen Osteosynthese erwogen werden [2].

Ein Goldstandard für das chirurgische Vorgehen existiert nicht. Das Spektrum reicht von der befundadaptierten Revisionschirurgie (Operationen nach Klinik, Paraklinik und Mikrobiologie) bis hin zur so genannten Etappenlavage, bei der die Patienten in einem festen Zeitrhythmus (alle $48 \mathrm{~h}$ ) lokal so lange revidiert werden, bis aus den intraoperativ gewonnenen mikrobiologischen Proben kein Keimnachweis mehr gelingt.

Im eigenen Vorgehen wird ein 3-Eingriffs-Modell bevorzugt. Nach der ersten Operation mit möglichst radikaler Infektherdsanierung („Ro-Resektion“) erfolgt geplant nach etwa 1 Woche ein weiterer Eingriff quasi zur Erfolgskontrolle. In Abhängigkeit vom dann erhobenen Befund wird ggf. die Indikation zu einer weiteren Operation, wiederum nach etwa 1 Woche gestellt.

Die Defektdeckung während dieser Sanierungsphase kann mit unterschiedlichen Maßnahmen gewährleistet werden. Das Spektrum reicht vom Feuchtverband mit $\mathrm{NaCl}$ o,9\% bis hin zur Vakuumversiegelung. Niemals ist jedoch der primäre Wundverschluss zu erzwingen, durch welchen das Gewebe noch zusätzlich kompromittiert werden kann.

\section{Schritt 2: Weichteilrekonstruktion}

Sie ist im Anschluss an die Infektberuhigung vordringlich anzustreben, aus dem Wissen heraus, dass intakte Weichteile die Voraussetzung für eine erfolgreiche Knochenrekonstruktion sind.

\begin{tabular}{|c|c|}
\hline $\begin{array}{l}\text { Knochendefekt- } \\
\text { größe }(\mathrm{cm})\end{array}$ & $\begin{array}{l}\text { Knochenrekon- } \\
\text { struktionsverfahren }\end{array}$ \\
\hline$\leq 3$ & $\begin{array}{l}\text { Spongiosaplastik } \pm \text { Kno- } \\
\text { chenwachstumsfaktoren }\end{array}$ \\
\hline$>3$ & Segmenttransport \\
\hline
\end{tabular}

Die chirurgischen Maßnahmen reichen von der Meshgraft-Plastik bis hin zu ausgedehnten mikrovaskularisierten Muskellappen. Nach Heppert et al. [1] ist die Methode der Weichteilrekonstruktion von folgenden Kriterien abhängig:

- geplante oder vorhandene Osteosynthese,

- Lokalisation und Ausmaß des Weichteildefekts,

- Durchblutungssituation und

- Compliance des Patienten.

\section{Schritt 3: Knochenrekonstruktion}

Ist ein intakter Weichteilmantel wiederhergestellt kann, mitunter auch überlappend, die Knochenrekonstruktion erfolgen, für welche ebenfalls eine weite Palette operativer Maßnahmen zur Verfügung steht. Als Faustregel kann die in $\mathbf{0}$ Tab. 1 dargestellte Beziehung zwischen dem Ausmaß des Knochendefekts und der Verfahrenswahl gelten:

\section{Stabilisierungsmaßnahmen}

Die mechanische Stabilität der befallenen Areale ist während der gesamten Behandlung gefordert, da bekannt ist, dass Instabilität einen Infekt unterhalten oder wieder anfachen kann. Auch hier steht ein breites Spektrum an Möglichkeiten zur Verfügung, als wesentlichste Maßnahme kann jedoch die Stabilisierung mittels Fixateur externe angesehen werden. Schmidt et al. [5] empfahlen den Gebrauch des Ilisarov-Fixateurs insbesondere dann, wenn weitreichende Rekonstruktionen am Knochen absehbar notwendig werden [5].

\section{Fazit für die Praxis}

Die Kenntnis der 3 Säulen der Therapie ist die Basis für die erfolgreiche Behandlung von Knocheninfekten:

- Chirurgie
Trauma Berufskrankh 2010 - 12 [Suppl 4]:472-474

DOI 10.1007/s10039-010-1639-2

(C) Springer-Verlag 2010

A.H. Tiemann · M. Steen · G.O. Hofmann

Infektsanierung und

Defektdeckung. Algorithmus und Reihenfolge chirurgische Strategie

\section{Zusammenfassung}

Infektionen des Knochens und des Skelettsystems zählen in der orthopädischen/unfallchirurgischen Praxis zu den schwerwiegenden Komplikationen. Nur die konsequente, zielfokussierte Therapie, basierend auf der chirurgischen Sanierung, dem Einsatz von Antibiotika (systemisch/lokal) sowie der Kenntnis und Anwendung adjuvanter Maßnahmen (z. B. hyperbare Oxygenierung, gezielte Applikation von Spurenelementen usw.), kann zum Erfolg, d. h. zur Infektberuhigung führen. Gerade die korrekte Reihenfolge der chirurgischen Maßnahmen auf dem Weg von der Resektion der befallenen Knochen und Weichteile bis zur erfolgreichen Rekonstruktion dieser Strukturen ist von großer Bedeutung. Die Kenntnis der speziellen Algorithmen ist eine Conditio sine qua non.

\section{Schlüsselwörter}

Orthopädische/unfallchirurgische Praxis . Knocheninfektion - Weichteilinfektion . Infektsanierung · Therapiealgorithmus

\section{Eradicating infection and closing a defect. Algorithm and sequence - surgical strategy}

\section{Abstract}

Bone infections are the most dramatic complications in orthopedic/trauma surgery. The successful treatment is based on the surgical treatment of the infection site combined with the use of antibiotics (systemically and locally) as well as on the use of adjuvant methods (i.e. hyperbaric oxygenation, application of trace elements). Especially the correct order in which the individual surgical procedures are performed from resection of the infected bone and the surrounding soft tissue to the reconstruction of bone and soft tissue is most important. It is a must that the treating surgeon knows about the algorithm to follow for the particular surgical steps.

\section{Keywords}

Orthopedic/trauma surgery - Bone infection . Soft tissue infection - Infection therapy .

Therapeutic algorithm 
- Antibiotika

- Adjuvanzien

Die chirurgische Infektsanierung ist die Grundlage der Behandlung.

Insuffiziente Chirurgie wird nicht durch den Einsatz von Antibiotika und adjuvanten Maßnahmen ausgeglichen.

Die Kenntnis und Beachtung des Therapiealgorithmus sind unabdingbar:

- Schritt 1: Infektberuhigung an Knochen und Weichteilen

- Schritt 2:Weichteilrekonstruktion

- Schritt 3 (individuell überlappend mit Schritt 2): Knochenrekonstruktion

\section{Korrespondenzadresse}

\section{PD Dr. A.H. Tiemann}

Abteilung für Septische

und Rekonstruktive Chirurgie, Klinik für

Unfall- und Wiederherstellungschirurgie,

BG-Kliniken Bergmannstrost Halle,

Merseburgerstraße 165, 06112 Halle, Saale

andreas.tiemann@bergmannstrost.com

Interessenkonflikt. Keine Angaben

\section{Literatur}

1. Heppert V, Glatzel U, Wentzensen A (2004) Postoperative und bakterielle Osteitis. Orthopäde 33:316-326

2. Hofmann GO (2004) Infektionen der Knochen und Gelenke. Urban \& Fischer, München, ISBN 3-43723400-5

3. Kälicke T, Kutscha-Lissberg F, Frangen TM et al (2004) Pathophysiologie der posttraumatischen Osteitis. Orthopäde 33:404-411

4. Schmidt HGK, Kranz HW, Siebert CH (1997) Die Behandlung von langstreckigen Infekt-Defekt-Pseudarthrosen mit Segmenttransport im Ringfixateur. Osteosyn Intern 5:212-220

5. Schmidt HGK, Wurm M, Halder D, Grosser V (2002) Verlängerungstechniken und Möglichkeiten des Segmenttransportes im Ringsystem nach llizarov. Trauma Berufskrankh 4:413-426

6. Tiemann AH, Schmidt HGK, Braunschweig R, Hofmann GO (2009) Strategies for the analysis of osteitic bone defects at the diaphysis of long bones. Strategies Trauma Limb Reconstr 4(1):13-18 